\title{
The Generalized wH Value
}

\author{
Tobias Hiller \\ Department Microeconomics, University Leipzig, Leipzig, Germany \\ Email: hiller@wifa.uni-leipzig.de
}

Received 29 December 2013; revised 17 January 2014; accepted 31 January 2014

Copyright (C 2014 by author and Scientific Research Publishing Inc.

This work is licensed under the Creative Commons Attribution International License (CC BY).

http://creativecommons.org/licenses/by/4.0/

(c) (i) Open Access

\section{Abstract \\ In this note, we generalize the $w H^{\text {Sh }}$ value [1] [2] for CO values.}

\section{Keywords}

\section{Cooperative Game Theory, Hierarchies, $w^{\text {Sh }}$ Value, Axiomatization}

\section{Introduction}

In this note, we generalize the $w H^{\text {Sh }}$ value [1] [2] for efficient values for TU (transferable utility) games with a cooperation structure (graph on the set of players; $\mathrm{CO}$ games and $\mathrm{CO}$ value) like the Myerson value [3], the position value [4], and the average tree solution [5]. This value determines payoffs in weighted hierarchical games. Modelling hierarchies in games of cooperative game theory is one step to analyze firms in the framework of cooperative game theory. A first step for this was done by Kalai and Samet [6] using ordered partitions and weights to model hierarchies. Another approach comes from Winter [7] using a sequence of bargaining components [8] to model hierarchies. These values can model levels in the sense of Lazear and Rosen [9], Carmichael [10], and Prendergast [11]. The problem of both approaches is that they could not model a clear manager-subordinate structure. Such a structure is a main element of hierarchies in firms [12] [13]. Another approach to model hierarchies might be games with an undirected graph/network on the set of players [3]-[5]. The problem is: all players are symmetrical in the graph; all players are in one level. The paper by van den Brink [14] uses a directed graph/permission structure on the set of players to analyze the effects of hierarchies on players' payoffs. The basic idea is that a player needs approval from all predecessors. The approach was outlined in Gilles, Owen, and van den Brink [15]. The axiomatization is carried out by van den Brink and Gilles [16].

In the modeling of van den Brink [14], single dominance relationships, i.e. the influence of a predecessor on the direct successors, are equally strong. It is, however, quite a plausible assumption that the relations among the players can have different levels of strength (e.g. they account for different leadership styles). This possibility is especially necessary when mapping corporate hierarchies. With this in mind, Casajus, Hiller and Wiese [1] [2] 
introduce the $w H^{S h}$ value. The authors use a weighted directed graph to model hierarchies. The basic idea for the $w H^{S h}$ value includes two elements. In order to create the output, all players work symmetrically together. As a first step, the result generated is distributed to the players according to the Shapley value [17]. In a second step, the weighted hierarchy reallocates a certain fraction of these payoffs. A player $i$ on the lowest hierarchical level gives the fraction $w_{i}$ from his Shapley payoff to his direct predecessor $j$. Player $j$ gives the fraction $w_{j}$ from all (gross) payoffs he receives (his Shapley payoff and the fractions from his successors) to his direct predecessor, etc. The weighted hierarchy has only allocation effects.

This is our starting point. We modify the $w H^{\text {Sh }}$ value in order to model the coordinating tasks of hierarchies. Within the framework of cooperative game theory, this is a new development starting from the papers done by Gilles, Owen, and van den Brink [15], van den Brink and Gilles [16], van den Brink [19], and van den Brink [14].

The paper is outlined as follows. In Section 3, we generalize the $w H^{\text {Sh }}$ value. Now the players do not work symmetrically together in order to create the output (first step). The problem of coordination of players is taken into account. The player who coordinates the other players is honored, if the coordination yields cooperation benefits. This approach is based on Hiller [18]. Section 4 concludes the paper. The paper starts with some preliminaries.

\section{Preliminaries}

A TU game is a pair $(N, v) . \quad N=\{1,2, \cdots, n\}$ is the set of players. The coalitional function $v$ assigns every subset $K$ of $N$ a certain worth $v(K)$ reflecting the economic abilities of $K$ i.e. $v: 2^{N} \rightarrow \mathrm{R}$ such that $v(\varnothing)=0$. A game $(N, v)$ is called symmetric if a function $f: N \rightarrow \mathrm{R}$ exists such that $v(K)=f(|K|)$ for all nonempty sets $K \subseteq N$, where $|K|$ denotes the cardinality of $K$. A symmetric game is called monotone if $v(T)<v(S)$, for all $T \subset S, T, S \subseteq N ; f(|K|)$ is increasing.

A TU value is an operator $\phi$ that assigns pay off vectors to all games $(N, v)$. One important value is the Shapley value. In order to calculate the players' payoffs, rank orders $\rho$ on $N$ are used. They are written as $\left(\rho_{1}, \cdots, \rho_{n}\right)$ where $\rho_{1}$ is the first player in the order, $\rho_{2}$ the second player etc. The set of the orders is denoted by $R O(N) ; n$ ! rank order sexist. The set of players before $i$ in rank order $\rho$ and player $i$ is called $K_{i}(\rho)$. Thus, given a player $i$ and a rank order $\rho$, the marginal contribution of $i$ is defined by $M C_{i}^{\rho}(v):=v\left(K_{i}(\rho)\right)-v\left(K_{i}(\rho) \backslash\{i\}\right)$. The Shapley value is the average of the marginal contributions taken over all rank orders of the players $[17]$ :

$$
S h_{i}(N, v)=\frac{1}{n !} \sum_{\rho \in R O(N)} M C_{i}^{\rho}(v) .
$$

In this note, we use one example to demonstrate the calculations.

Example 1 We assume a game with $N=\{1,2,3\}$ and

$$
v(K)=\left\{\begin{array}{lc}
2, & |K|=1 \\
10, & |K|=2 \\
15, & |K|=3 \\
0, & \text { else. }
\end{array}\right.
$$

This game is symmetric and monotone. The $S h_{i}(N, v)$ are calculated by:

$$
\begin{aligned}
& S h_{1}(N, v)=\frac{1}{3 !}(\underset{(1,2,3)}{2}+\underset{(1,3,2)}{2}+\underset{(2,, 1,3)}{8}+\underset{(2,3,1)}{5}+\underset{(3,1,2)}{8}+\underset{(3,2,2)}{5})=5 \\
& S h_{2}(N, v)=S h_{3}(N, v)=5 .
\end{aligned}
$$

As in Gilles, Owen, and van den Brink [15], van den Brink and Gilles [16], van den Brink [19], van den Brink [14], and Casajus, Hiller and Wiese [1] [2], the permission structure is a mapping $S: N \rightarrow 2^{N}$. It maps to each player $i$ those players that are direct successors of $i . S$ can be interpreted as directed graph [20]. $S(i)$ identifies the direct successors of $i$ with $i \notin S(i)$. The players in $S^{-1}(i)=\{j \in N: i \in S(j)\}$ are called direct predecessors of $i ; S^{-1}(K)=\bigcup_{i \in K} S^{-1}(i)$. 
As usual in the literature, it is assumed that the hierarchy has a tree structure [21] [22]. These structures satisfy two conditions:

- there is one player $i_{0} \in N$, such that $S^{-1}\left(i_{0}\right)=\varnothing$ and $\hat{S}\left(i_{0}\right)=N \backslash\left\{i_{0}\right\}$ and

- for every player $i \in N \backslash\left\{i_{0}\right\}$ we have $\left|S^{-1}(i)\right|=1$.

In a tree structure, a path $T$ in $N$ from $i$ to $j$ is a sequence of players $T(i, j)=\left\langle r_{0}, r_{1}, \cdots, r_{k-1}, r_{k}\right\rangle$ with $i=r_{0}, j=r_{k}$ and $S\left(r_{\ell}\right)=r_{\ell+1}$ for all $\ell=0, \cdots, k-1$. A path can be interpreted as a chain of commands between $i$ and $j$, whereby $i$ is a predecessor of $j$. The set of successors of $i$ is denoted by $\hat{S}(i):=\{j \in N \backslash\{i\}:$ there is a path from $i$ to $j\}$. Analogously, we denote the set of $i$ 's predecessors by $\hat{S}^{-1}(i):=\{j \in N \backslash\{i\}:$ there is a path from $j$ to $i\}$.

Beyond this hierarchy $S$, weighted relations between players are considered. The vector $w: N \rightarrow \mathrm{R}$ maps to every player $i$ a weight $w_{i}, 0 \leq w_{i} \leq 1$. For $i_{0}$ we have $w_{i_{0}}=0$. If a vector maps all players the same weight $\bar{w}$, except $i_{0}$, i.e. $w_{i}=w_{j}=\bar{w}$ for all $i, j \in N \backslash\left\{i_{0}\right\}$, we also denote the vector by $\bar{w}$. With $w[K]$ we denote a vector that maps to all $i \in K$ the weight zero, $w[K]_{i}=0$. The players $j \in N \backslash K$ retain their initial weights, $w[K]_{j}=w_{j}$. To simplify your notation, we write $w[i, j, \cdots]$ instead of $w[\{i, j, \cdots\}]$.

A weighted hierarchical game is a tuple $(N, v, S, w)$. A weighted hierarchical value is an operator $\varphi$. The $w H^{S h}$ value is one weighted hierarchical value. All players $j$, with $j \in \hat{S}^{-1}(g)$ respectively all players in the path $T\left(i_{0}, g\right)$, get a fraction of $g$ 's Shapley payoff. Any player $i \in N$ gets from $g$ 's Shapley payoff the fraction [1] [2]:

$$
f_{i}(S, w, g)=\left\{\begin{array}{lc}
{\left[1-w_{i}\right] \prod_{l \in \hat{S}(i), l \in T\left(i_{0}, g\right)} w_{l},} & i \in T\left(i_{0}, g\right), \\
0, & \text { else. }
\end{array}\right.
$$

With this formula, the wH-payoff of player $i \in N, w H_{i}^{S h}(N, v, S, w)$, is determined by [1] [2]:

$$
w H_{i}^{S h}(N, v, S, w)=\sum_{j=1}^{n} f_{i}(S, w, j) \cdot S h_{j}(N, v) .
$$

Example 2 In addition to example 1, we assume $S(1)=S(2)=\varnothing, \quad S_{3}=\{1,2\}$, and $w\left(\frac{1}{4}, \frac{1}{3}, 0\right)$. Figure 1 illustrates the weighted hierarchy. For the players' $w H^{\text {Sh }}$ payoffs, we have:

$$
\begin{aligned}
w H_{3}^{S h}(N, v, S, w)= & f_{3}(S, w, 1) \cdot S h_{1}(N, v)+f_{3}(S, w, 2) \cdot S h_{2}(N, v) \\
& +f_{3}(S, w, 3) \cdot S h_{3}(N, v) \\
= & \frac{1}{4} \times 5+\frac{1}{3} \times 5+1 \times 5=7 \frac{11}{12} \\
w H_{1}^{S h}(N, v, S, w)= & 3 \frac{3}{4} \\
w H_{2}^{S h}(N, v, S, w)= & 3 \frac{1}{3} .
\end{aligned}
$$

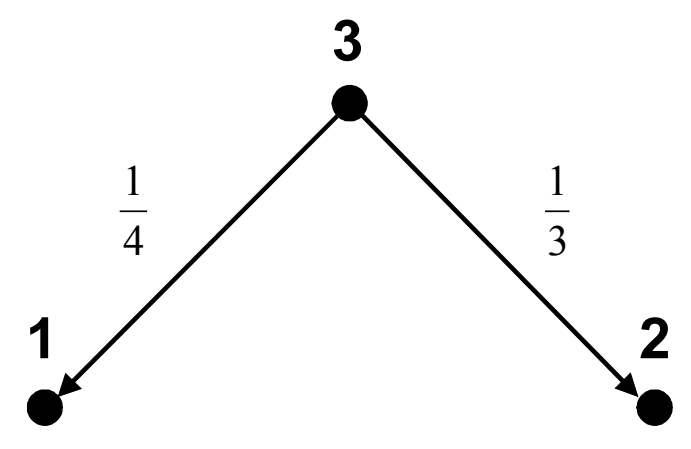

Figure 1. Weighted hierarchical game. 
Casajus, Hiller and Wiese [1] [2] present eight axioms for the axiomatization of the $w H^{S h}$ value. They can be categorized into two groups. The first four axioms-additivity (A), efficiency (E), weak symmetry (WS), weak null player $(\mathbf{W N})$ - are modified Shapley axioms. They ensure that the Shapley payoffs results if $\bar{w}=w[N]$. The last four axioms - gross net (GN), splitting (SP), isolation (IS), independency (ID) — control the reallocation from the bottom to the top of the hierarchy.

Axiom 3 (A) For all coalitional functions $v$ and $z$,

$$
\varphi_{i}(N, v, S, w)+\varphi_{i}(N, z, S, w)=\varphi_{i}(N, v+z, S, w), \quad i \in N .
$$

A players' payoff in the game $(N, v+z, S, w)$ should be the sum of the payoffs he achieved in two separate games $(N, v, S, w)$ and $(N, z, S, w)$.

Axiom 4 (E) It holds

$$
\sum_{i \in N} \varphi_{i}(N, v, S, w)=v(N) .
$$

The worth $v(N)$ is distributed among the players.

Definition 5 (S) Players $i, j \in N$ are called symmetric, if $v(K \cup\{i\})-v(K)=v(K \bigcup\{j\})-v(K)$ for all $K \subseteq N, \quad i, j \notin K$.

I.e., both players $i$ and $j$ have the same marginal contributions to coalitions that do not contain them.

Axiom 6 (WS) If $i, j \in N$ are symmetric then

$$
\varphi_{i}(N, v, S, w[N])=\varphi_{j}(N, v, S, w[N])
$$

This axiom says that symmetric players get the same payoffs if all weights are zero.

Definition 7 (N) Player $i \in N$ is a null player, if $v(K \cup\{i\})=v(K)$ for all $K \subseteq N$.

Axiom 8 (WN) If $i \in N$ is a null player then $\varphi_{i}(N, v, S, w[N])=0$.

If all weights are zero, a null player gets the payoff zero.

Axiom 9 (GN) For the relation between gross and net payoffs, the equation

$$
\varphi_{i}(N, v, S, w)=\left(1-w_{i}\right) \cdot \varphi_{i}(N, v, S, w[i])
$$

holds.

The gross payoff $w H_{i}(N, v, S, w[i])$ is the value that $i$ would receive if he did not have to pay anything to his direct predecessor. If this value is multiplied with $\left(1-w_{i}\right)$, the net pay off results.

Axiom 10 (SP) For the relation between player $i$ 's pay off and player $j$ 's payoff, $j \in S(i)$, the following equation holds:

$$
\varphi_{i}(N, v, S, w[i])-\varphi_{i}(N, v, S, w[i, j])=\varphi_{j}(N, v, S, w[j])-\varphi_{j}(N, v, S, w) .
$$

This axiom says that reducing the weight between $i$ and $j$ to zero ( $w_{j}$ is changed to zero), reduces the gross payoff of player $i$ (left-hand side of equation) in the same way as it raises the payoff of player $j$ (right-hand side).

Axiom 11 (IS) For any player $i$ holds:

$$
\varphi_{i}(N, v, S, w[\{i\} \cup S(i)])=\varphi_{i}(N, v, S, w[N])
$$

This axioms states that player $i$ get the same payoff in two cases. In the first case, his weight is zero and the weights of his direct successors are zero too. In the second case, all players' weights are zero.

Axiom 12 (ID) If a player participates in two weighted hierachical games $(N, v, S, w)$ and $\left(N, v, S, w^{\prime}\right)$ with $w_{j}=w_{j}^{\prime}$, for all $j \in \hat{S}(i) \cup\{i\}$, then

$$
\varphi_{i}(N, v, S, w)=\varphi_{i}\left(N, v, S, w^{\prime}\right) .
$$

The games $(N, v, S, w)$ and $\left(N, v, S, w^{\prime}\right)$ have the same set of players $N$, the same coalitional function $v$ and the same hierarchy $S$. Further, the weight of $i$ and the weights of all players $\hat{S}(i)$ are the same in both games. The axiom states that $i$ s payoff should be the same in both games.

Now we will introduce some preliminaries for CO games (for a literature review on CO games see Slikker and van den Nouweland [23]). This games account for coordination tasks of managers in firms. The set of possible pair wise links between players is called $L^{N}=\{\{i, j\}: i, j \in N, i \neq j\}$, whereat $\{i, j\}$ and $\{j, i\}$ respec- 
tively (or $i j$ and $j i$ respectively) is the direct link between $i$ and $j$. A cooperation structure on $N$ is a graph $(N, L)$ with $L \subseteq L^{N}$. A CO game $(N, v, L)$ is a game $(N, v)$ together with a cooperation structure $(N, L)$. A CO value is an operator $\psi$ that assigns payoff vectors to all $\mathrm{CO}$ games. The most popular $\mathrm{CO}$ values are the Myerson value [3], the position value [4], and the average tree solution [5]. From $S$ we construct $L_{S}$ in the following way: $L_{S}=\{i j: i \in S(j)\}$ for all $i, j \in N$. The tree structure is inherited from $S$ to $L_{S}$ Hence, we have a CO game $\left(N, v, L_{S}\right)$.

Example 13 In our example, we have $L_{S}=\{13 ; 23\}$.

We will exemplify the CO generalization of the $w H^{S h}$ value with the Myerson value. To calculate the Myerson payoffs, we need some more preliminaries. The graph partitions the set of players into components $C_{1}, \cdots, C_{k}$. This partition is denoted by $N \backslash L$. Connected (directly or indirectly) players are in one component. Each player is in one component; $C_{i} \cap C_{j}=\varnothing, \quad i \neq j, \quad N=\bigcup C_{j} . N \backslash L(i)$ denotes the component containing player $i$. Now, we can introduce the restricted coalitional function $\left.v\right|_{L}[3]$ :

$$
\left.v\right|_{L}(K):=\sum_{C \in K \backslash L} v(C) \text { for all } K \subseteq N .
$$

The worth of a coalition $K$ is the sum of the worth of its components. In the case of $|K \backslash L|=1$ we have $v(K)=\left.v\right|_{L}(K)$ for $K$. The Myerson payoff of player $i$ is determined by:

$$
M y_{i}(N, v, L)=S h_{i}\left(N,\left.v\right|_{L}\right) .
$$

Example 14 In our example we have

$$
\left.v\right|_{L_{S}}(K)= \begin{cases}2, & |K|=1 \\ 4, & K=\{1,2\} \\ 10, & K=\{1,3\}, K=\{2,3\} \\ 15, & |K|=3 \\ 0, & \text { else. }\end{cases}
$$

Hence, we obtain:

$$
\begin{aligned}
& M y_{3}\left(N, v, L_{S}\right)=\frac{1}{3 !}(\underbrace{11}_{(1,2,3)}+\underset{(1,3,2)}{8}+\underset{(2,1,3)}{11}+\underset{(2,3,1)}{8}+\underset{(3,1,2)}{2}+\underset{(3,2,1)}{2})=7 \\
& \operatorname{My}_{1}\left(N, v, L_{S}\right)=M y_{2}\left(N, v, L_{S}\right)=4 .
\end{aligned}
$$

Since player 3 coordinates the other players, he gets a higher payoff.

\section{Generalization for CO Games}

In this section we generalize the $w H$ value for $\mathrm{CO}$ games. First, we will introduce an axiom that is used to simplify the original axiomatization [18]:

Axiom 15 (zero weights invariance $L$, ZWIL) If $w=w[N]$, then $\varphi_{i}(N, v, S, w)=\psi_{i}\left(N, v, L_{S}\right)$ for all $i \in N$.

This axiom says that the players get the payoff $\psi_{i}\left(N, v, L_{S}\right)$, if all weights are zero. $\psi$ could be any efficient $\mathrm{CO}$ value. Using axiom ZWIL, we have

Corollary 16 The $w H^{L}$ value is the unique weighted hierarchical value satisfying GN, SP, IS, ID, and ZWIL.

The formula to calculate the $w H^{L}$ payoff of player $i \in N$ is:

$$
w H_{i}^{L}(N, v, S, w)=\sum_{j=1}^{n} f_{i}(S, w, j) \cdot \psi_{j}\left(N, v, L_{S}\right) .
$$

Hence it is now possible to model coordinating tasks within the $w H$ framework.

Using the $M y$ value for $\psi$, we get the $w H^{M y}$ payoffs:

$$
w H_{i}^{M y}(N, v, S, w)=\sum_{j=1}^{n} f_{i}(S, w, j) \cdot M y_{j}\left(N, v, L_{S}\right)
$$


For our example, we obtain:

Example 17 In our example, we get the following $w H^{M y}$ payoffs:

$$
\begin{aligned}
w H_{3}^{M y}(N, v, S, w)= & f_{3}(S, w, 1) \cdot M y_{1}\left(N, v, L_{S}\right)+f_{3}(S, w, 2) \cdot M y_{2}\left(N, v, L_{S}\right) \\
& +f_{3}(S, w, 3) \cdot M y_{3}\left(N, v, L_{S}\right) \\
= & \frac{1}{4} \times 4+\frac{1}{3} \times 4+1 \times 7=9 \frac{1}{3} \\
w H_{1}^{M y}(N, v, S, w)= & 3 \\
w H_{2}^{M y}(N, v, S, w)= & 2 \frac{2}{3} .
\end{aligned}
$$

For our example, Table 1 shows the players' payoffs for the $w H^{S h}$ value and the $w H^{M y}$ value. It is easy to see that the $w H^{M y}$ value rewards player 3 since he coordinates players 1 and 2 .

Table 1. Payoffs for the example.

\begin{tabular}{cccc}
\hline & 1 & 2 & 3 \\
\hline$w H_{i}^{S h}$ & $3 \frac{3}{4}$ & $3 \frac{1}{3}$ & $7 \frac{11}{12}$ \\
$w H_{i}^{M y}$ & 3 & $2 \frac{2}{3}$ & $9 \frac{1}{3}$ \\
\hline
\end{tabular}

\section{Conclusion}

In this paper, we generalized the $w H^{S h}$ value for efficient $\mathrm{CO}$ values. The new value $w H^{L}$ takes into account the problem of coordination of players - one main task for managers in firms. Hence, the $w H^{L}$ approach is another step in modelling firms and hierarchies. In the first step, the worth $v(N)$ is divided among the players with respect to their productivity and their coordination tasks. In the second step, the weighted hierarchy reallocates a certain fraction of these payoffs. We hope that this value is an appropriate value for analyzing hierarchies in firms.

\section{References}

[1] Casajus, A., Hiller, T. and Wiese, H. (2009) Hierarchie und Entlohnung. Zeitschrift für Betriebswirtschaft, 79, 929-954. http://dx.doi.org/10.1007/s11573-009-0271-8

[2] Casajus, A., Hiller, T. and Wiese, H. (2012) Hierarchy and Wages. Working Paper.

[3] Myerson, R.B. (1977) Graphs and Cooperation in Games. Mathematics of Operations Research, 2, 225-229. http://dx.doi.org/10.1287/moor.2.3.225

[4] Borm, P., Owen, G. and Tijs, S. (1992) On the Position Value for Communication Situations. SIAM Journal on Discrete Mathematics, 5, 305-320. http://dx.doi.org/10.1137/0405023

[5] Herings, P.J.J., van der Laan, G. and Talman, D. (2008) The Average Tree Solution for Cycle-Free Graph Games. Games and Economic Behavior, 62, 77-92. http://dx.doi.org/10.1016/j.geb.2007.03.007

[6] Kalai, E. and Samet, D. (1987) On Weighted Shapley Values. International Journal of Game Theory, 16, $205-222$. http://dx.doi.org/10.1007/BF01756292

[7] Winter, E. (1989) A Value for Cooperative Games with Levels Structure of Cooperation. International Journal of Game Theory, 18, 227-240. http://dx.doi.org/10.1016/S0167-2681(01)00156-1

[8] Owen, G. (1977) Values of Games with a Priori Unions. In: Henn, R. and Moeschli, O. Eds., Essays in Mathematical Economics \& Game Theory, Springer Verlag, Berlin et al., 76-88.

[9] Lazear, E.P. and Rosen, S. (1981) Rank-Order Tournaments as Optimum Labor Contracts. Journal of Political Economy, 89, 841-864. www.jstor.org/stable/1830810 http://dx.doi.org/10.1086/261010

[10] Carmichael, L. (1983) Firm-Specific Human Capital and Promotion Ladders. Bell Journal of Economics, 14, 251258.www.jstor.org/stable/3003551 http://dx.doi.org/10.2307/3003551

[11] Prendergast, C. (1993) The Role of Promotion in Inducing Specific Human Capital Acquisition. Quarterly Journal of Economics, 108, 523-534.www.jstor.org/stable/2118343 
[12] Radner, R. (1992) Hierarchy: The Economics of Managing. Journal of Economic Literature, 30, 1382-1415. www.jstor.org/stable/2728063

[13] Meagher, K.J. (2001) The Impact of Hierarchies on Wages. Journal of Economic Behavior and Organization, 45, 441458. http://dx.doi.org/10.1016/S0167-2681(01)00156-1

[14] van den Brink, R. (2008) Vertical Wage Differences in Hierarchically Structured Firms. Social Choice and Welfare, 30, 225-243. http://dx.doi.org/10.1007/s00355-007-0230-7

[15] Gilles, R.P., Owen, G. and van den Brink, R. (1992) Games with Permission Structures: The Conjunctive Approach. International Journal of Game Theory, 20, 277-293. http://dx.doi.org/10.1007/BF01253782

[16] van den Brink, R. and Gilles, R.P. (1996) Axiomatizations of the Conjunctive Permission Value for Games with Permission Structure. Games and Economic Behavior, 12, 113-126. http://dx.doi.org/10.1006/game.1996.0008

[17] Shapley, L.S. (1953) A Value for N-Person Games. In: Kuhn, H.W. and Tucker, A.W., Eds., Contributions to the Theory of Games, Vol. 2, Princeton, 307-317.

[18] Hiller, T. (2011) A Note on Chi-Values. International Review of Economics, 58, 433-438. http://dx.doi.org/10.1007/s12232-011-0125-x

[19] van den Brink, R. (1997) An Axiomatization of the Disjunctive Permission Value for Games with a Permission Structure. International Journal of Game Theory, 26, 27-43. http://dx.doi.org/10.1007/BF01262510

[20] Bollobás, B. (2002) Modern Graph Theory. 3rd Edition, NewYork, Heidelberg et al. http://dx.doi.org/10.1016/S0167-2681(01)00156-1

[21] Radner, R. (1992) Hierarchy: The Economics of Managing. Journal of Economic Literature, 30, 1382-1415. www.jstor.org/stable/2728063

[22] Meagher, K.J. (2001) The Impact of Hierarchies on Wages. Journal of Economic Behavior and Organization, 45, 441458.

[23] Slikker, M. and van den Nouweland, A. (2001) Social and Economic Networks in Cooperative Game Theory, Boston et al. 